\title{
A Comparative Study of Auto-contouring Softwares in Delineation of Organs at Risk in Lung Cancer and Rectal Cancer
}

weijun chen

Zhejiang Provincial People's Hospital

Cheng Wang

University of South China

Wenming Zhan

Zhejiang Provincial People's Hospital

Yongshi Jia

Zhejiang Provincial People's Hospital

Fangfang Ruan

Hangzhou Medical College

Lingyun Qiu

Zhejiang Provincial People's Hospital

\section{Shuangyan Yang}

Shanghai Pneumology Hospital: Tongji University Affiliated Shanghai Pulmonary Hospital

Yucheng Li ( $\sim$ liyucheng0402@163.com)

Zhejiang Provincial People's Hospital ,Hangzhou https://orcid.org/0000-0002-1383-6574

\section{Research}

Keywords: Cancer of the lungs, Cancer of the rectum, Auto-contouring, Organs at risk

Posted Date: March 22nd, 2021

DOI: https://doi.org/10.21203/rs.3.rs-323597/v1

License: (c) (i) This work is licensed under a Creative Commons Attribution 4.0 International License. Read Full License

Version of Record: A version of this preprint was published at Scientific Reports on November 26th, 2021. See the published version at https://doi.org/10.1038/s41598-021-02330-y. 


\section{Abstract}

Background:Radiotherapy requires the target area and the organs at risk to be contoured on the CT image of the patient. During the process of organs-at-Risk (OAR) of the chest and abdomen, the doctor needs to contour at each CT image. The delineations of large and varied shapes are time-consuming and laborious.

This study aims to evaluate the results of two automatic contouring software on OAR definition of CT images of lung cancer and rectal cancer patients.

Methods: The CT images of 15 patients with rectal cancer and 15 patients with lung cancer were selected separately, and the organs at risk were outlined by the same experienced doctor as references, and then the same datasets were automatically contoured based on AiContour ${ }^{\circledR \odot}$ (Manufactured by Linking MED, China) and Raystation ${ }^{\circledR \odot}$ (Manufactured by Raysearch, Sweden) respectively. Overlap index (OI), Dice similarity index (DSC) and Volume difference ( $\left.D_{\vee}\right)$ were evaluated based on the auto-contours, and independent-sample $t$-test analysis is applied to the results.

Results: The results of AiContour ${ }^{\circledR \odot}$ on OI and DSC were better than that of Raystation ${ }^{\circledR \odot}$ with statistical difference. There was no significant difference in $D_{V}$ between the results of two software.

Conclusions: With AiContour ${ }^{\circledR \odot}$, auto-contouring results of most organs in the chest and abdomen are good, and with slight modification, it can meet the clinical requirements for planning. With Raystation ${ }^{\circledR}$, auto-contouring results in most OAR is not as good as AiContour ${ }^{\circledR \odot}$, and only the auto-contouring results of some organs can be used clinically after modification.

\section{Introduction}

In recent years, the incidence of thoracic and abdominal tumors is getting higher and higher, and most are Lung and Rectum tumors. In order to improve the cure rate, about $70 \%$ of patients with malignant tumors need to receive radiation therapy. Radiotherapy has gradually become the second largest cancer treatment technology after surgery ${ }^{[1,2]}$. Radiotherapy requires the target area and the organs at risk to be contoured on the CT image of the patient. During the process of organs-at-Risk (OAR) of the chest and abdomen, the doctor needs to contour at each CT image. The delineations of large and varied shapes are time-consuming and laborious. Moreover, the OAR contours of the same patient by different doctors are subjectively different. If automatic contouring of organs at risk is implemented, the efficiency of the doctors can be improved, and the subjective differences of contours by different doctors can be reduced. The efficiency and consistency of treatment are of great significance ${ }^{[3-6]}$. With the development of science and technology, the accuracy and efficiency of radiation therapy need to be improved. From automatic contour softwares available on the market, we have selected AiContour ${ }^{(C)}$ (Linking MED, China) intelligent contouring system and Raystation $®(C)$ (Research, Sweden) automatic delineating system to analyze the results of shape similarity compared to the contour from experience doctor. Index (Dice Similarity Coefficient, DSC), overlap index (OI) and volume difference $\left(D_{v}\right)$ were evaluated to analyze the accuracy of automatic contouring of thoracic and abdominal organs. Finally, independent sample t-test proofreading was performed with SPSS.

\section{Materials And Methods}

\section{General Information}

We select 15 patients with lung cancer and 15 patients with rectal cancer who were treated in Zhejiang Province People's Hospital from March 2019 to November 2019 randomly. All patients were placed in supine position during CT simulation, and a thermoplastic masks and vacuum cushions were selected based on clinical needs. The body position is fixed, and 
the scanning slice thickness is $5 \mathrm{~mm}$. The patients' CT were selected as target images, and the data template libraries of the two software were used for automatic sketching.

\section{Software tools}

Using the Atlas Based segmentation function in Raystation $₫($ and the artificial intelligence automatic cloud delineating software AiContour ${ }^{(}($C. Raystation $(\subset)$ uses an algorithm based on interactive information to select template from the Atlas library. Contour the best matching case of CT, deform the outline of the case, and map the result of the deformation to the CT to be delineated to form an automatically delineated outline ${ }^{[7]}$. The AiContour ${ }^{\circledR}\left({ }^{C}\right.$ system is based on the segmentation network training of UNet ${ }^{[8]}$, forming recognition ability, so as to achieve the ability of automatic contour drawing. The target CT images automatically contour by the two sets of software are from the scanning of the largeaperture four-dimensional CT simulator (GE , America).

\section{Contour Content}

For lung cancer patients, pancreas, spleen, stomach, liver, esophagus, heart, left lung, right lung, and spinal cord were selected as the contouring OARs. For rectal cancer patients, the OARs are left kidney, right kidney, spinal cord, left femoral head, right femoral head, left leg bone, right leg bone, pelvis, rectum, and bladder. The organs at risk manually contoured by the doctor on the Raystation $(@)$ planning system was selected as the standard, and the results of the automatic contours created by two auto-contouring software were respectively imported into the planning system and compared.

\section{Data analysis}

Using the OAR outline manually contoured by the experienced doctor as a reference, the results from two software were assessed with the cross-reference OI, shape similarity index DSC, and volume difference Dv respectively.

$\mathrm{Ol}=\left(\mathrm{V}_{\mathrm{a}} \cap \mathrm{V}_{\mathrm{m}}\right) / \mathrm{V}_{\mathrm{m}} \quad \nabla 1 \mathrm{1}$

$\mathrm{DSC}=2\left(\mathrm{~V}_{\mathrm{a}} \cap \mathrm{V}_{\mathrm{m}}\right) /\left(\mathrm{V}_{\mathrm{a}}+\mathrm{V}_{\mathrm{m}}\right) \quad \nabla 2 \bigotimes$

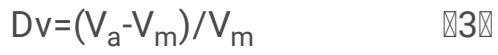

Among them, Va represents the volume $\left(\mathrm{cm}^{3}\right)$ automatically contoured by the software, and $\mathrm{V}_{\mathrm{m}}$ represents the volume $\left(\mathrm{cm}^{3}\right)$ manually contoured by the doctor. Among them, the closer the OI index and the DSC index are to 1, and the closer the $D_{v}$ value is to 0 , the better the delineating result.

\section{Statistical methods}

SPSS 23.0 was used to perform independent sample $t$-test statistical analysis on the results contoured by the two software. The difference was statistically significant with $P<0.05$, and the analysis was plotted by using Origin 8.0. (As shown in Figures 1 and 2)

\section{Results}

We have analyzed OI, DSC, and $D_{v}$ for patients with lung cancer and rectal cancer. 
The results of OAR delineation with AiContour ${ }^{\circledR}(\subset)$ in Lung cancer cases show that the average values of OI and DSC delineations of most organs are better than 0.8 , and the mean $D_{v}$ of most delineated organs are $<0.1$. Among them, the right lung has the best OI, DSC and Dv, and the mean OI is $>0.97$. The average DSC is $>0.98$, and the average $D_{v}$ is -0.003 . The worst OI and DSC mean is the pancreas. The mean OI is less than 0.67 , the mean DSC is less than 0.66 , and the biggest difference between $D_{v}$ is the esophagus, and the mean $D_{v}$ is greater than 0.3 . Compared with lung cancer, the OAR contour of rectal cancer is more stable. Both $\mathrm{OI}$ and DSC are greater than 0.8 , and $D_{v}$ are less than 0.1 . In the spinal cord delineation of patients with lung and rectal cancer, rectal cancer delineation results are better in terms of various values.

Raystation ${ }^{\circledR}$ ( $\subset$ results in lung cancer patients' OAR delineation show that the delineation results of different organs are quite diverse, and the delineation results of some organs are poor. Among DSC and OI, the best results of OI and DSC are left and right lungs. The mean OI and DSC values are both greater than 0.95 . The best result of $D_{v}$ is the right kidney. The mean value of $D_{v}$ is 0.003 . The lowest $\mathrm{Ol}$ and DSC is the pancreas, with Ol less than 0.32 , DSC less than 0.28 , and the worst $D_{v}$ is the bladder, with an average $D_{v}>0.84$. The interval of some data of Raystation® $@$ is relatively large. It may be that in some patients, the library does not have a good CT case with the CT to be outlined, and the deformation registration ability is insufficient, resulting large differences in the results. The comparison results of the two softwares are shown in Tables 1 and 2.

Table 1

Comparison of OI and DSC for OAR automatic delineation of rectal cancer by two software

\begin{tabular}{|c|c|c|c|c|c|c|c|c|c|c|c|}
\hline & & $\begin{array}{l}\text { Left } \\
\text { Kidney }\end{array}$ & $\begin{array}{l}\text { Right } \\
\text { Kidney }\end{array}$ & spinal & $\begin{array}{l}\text { Left } \\
\text { Femur } \\
\text { head }\end{array}$ & $\begin{array}{l}\text { Right } \\
\text { Femur } \\
\text { head }\end{array}$ & $\begin{array}{l}\text { left } \\
\text { Leg } \\
\text { bone }\end{array}$ & $\begin{array}{l}\text { Right } \\
\text { Leg } \\
\text { bone }\end{array}$ & pelvis & rectum & bladder \\
\hline \multirow[t]{2}{*}{ 이 } & AiContour & 0.971 & 0.964 & 0.972 & 0.936 & 0.945 & 0.963 & 0.969 & 0.942 & 0.870 & 0.836 \\
\hline & Raystation & 0.492 & 0.631 & 0.715 & 0.896 & 0.904 & 0.891 & 0.884 & 0.881 & 0.528 & 0.592 \\
\hline $\mathrm{t}$ & & 7.630 & 5.929 & 4.465 & 1.867 & 1.810 & 3.391 & 3.743 & 3.617 & 4.779 & 2.284 \\
\hline $\mathrm{P}$ & & 0 & 0 & 0.001 & 0.072 & 0.081 & 0.003 & 0.002 & 0.002 & 0 & 0.030 \\
\hline \multirow[t]{2}{*}{ DSC } & AiContour & 0.952 & 0.956 & 0.943 & 0.926 & 0.937 & 0.975 & 0.974 & 0.957 & 0.874 & 0.805 \\
\hline & Raystation & 0.492 & 0.632 & 0.687 & 0.878 & 0.883 & 0.877 & 0.870 & 0.895 & 0.439 & 0.448 \\
\hline $\mathrm{t}$ & & 8.198 & 6.042 & 5.156 & 3.808 & 3.262 & 5.777 & 5.762 & 5.907 & 7.599 & 4.057 \\
\hline $\mathrm{P}$ & & 0 & 0 & 0 & 0.001 & 0.003 & 0 & 0 & 0 & 0 & 0 \\
\hline \multirow[t]{2}{*}{ Dv } & AiContour & 0.039 & 0.017 & 0.061 & 0.022 & 0.017 & -0.026 & -0.011 & -0.031 & -0.012 & 0.081 \\
\hline & Raystation & -0.015 & 0.003 & 0.056 & 0.041 & 0.049 & 0.036 & 0.037 & -0.033 & 0.390 & 0.843 \\
\hline $\mathrm{t}$ & & 0.577 & 0.217 & 0.051 & 0.554 & -1.081 & -1.284 & 0.020 & 0.083 & -2.583 & -1.473 \\
\hline$P$ & & 0.569 & 0.831 & 0.960 & 0.584 & 0.289 & 0.219 & 0.984 & 0.935 & 0.021 & 0.163 \\
\hline
\end{tabular}


Table 2

Comparison of OI and DSC for OAR automatic delineation of lung cancer by two software

\begin{tabular}{|c|c|c|c|c|c|c|c|c|c|c|}
\hline & & pancreas & spleen & stomach & liver & esophagus & heart & Lung & $\begin{array}{l}\text { R } \\
\text { Lung }\end{array}$ & spinal \\
\hline \multirow[t]{2}{*}{ 이 } & AiContour & 0.664 & 0.916 & 0.836 & 0.972 & 0.860 & 0.916 & 0.973 & 0.979 & 0.944 \\
\hline & Raystation & 0.315 & 0.670 & 0.459 & 0.886 & 0.573 & 0.816 & 0.959 & 0.955 & 0.671 \\
\hline $\mathrm{t}$ & & 6.094 & 4.121 & 7.167 & 5.414 & 4.558 & 2.766 & 1.972 & 4.155 & 7.972 \\
\hline$P$ & & 0 & 0.001 & 0 & 0 & 0 & 0.010 & 0.059 & 0 & 0 \\
\hline \multirow[t]{2}{*}{ DSC } & AiContour & 0.650 & 0.920 & 0.832 & 0.965 & 0.751 & 0.925 & 0.977 & 0.981 & 0.896 \\
\hline & Raystation & 0.277 & 0.492 & 0.471 & 0.800 & 0.398 & 0.858 & 0.956 & 0.960 & 0.583 \\
\hline$t$ & & 7.139 & 8.804 & 6.899 & 14.214 & 10.194 & 2.486 & 3.476 & 4.345 & 9.565 \\
\hline$P$ & & 0 & 0 & 0 & 0 & 0 & 0.019 & 0.002 & 0 & 0 \\
\hline \multirow[t]{2}{*}{ Dv } & AiContour & 0.066 & -0.010 & 0.010 & 0.015 & 0.301 & -0.019 & -0.010 & -0.003 & 0.107 \\
\hline & Raystation & 0.544 & 0.824 & -0.033 & 0.222 & 0.832 & -0.109 & 0.007 & -0.010 & 0.355 \\
\hline$t$ & & -1.292 & -2.794 & 0.514 & -4.029 & -2.278 & 2.224 & -1.078 & 1.309 & -2.179 \\
\hline$P$ & & 0.214 & 0.014 & 0.614 & 0.001 & 0.037 & 0.034 & 0.290 & 0.204 & 0.046 \\
\hline
\end{tabular}

\section{Discussions}

In the design of radiation treatment plans, the accuracy of organ at risk contour often affects the dose distribution in patients and affects the actual target area and the dose of organ at risk, then impacts the treatment quality ultimately ${ }^{\text {[9- }}$ 10]. Under the development of science and technology, the automatic delineating performance has also been continuously optimized and improved. Due to the high precision of the automatic delineating technology, doctors can use it for clinical purpose with only slight modifications, reducing unnecessary workload for clinicians. It also greatly improves the treatment efficiency.

During the manual delineation of the organs in the chest and abdomen, the time of manual delineation of one patient's

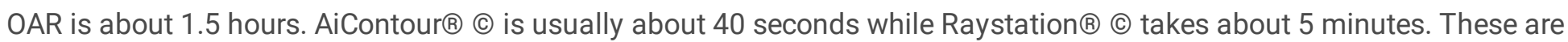
consistent with the results of Lustberg $T$ et al's studies ${ }^{[11]}$, the median time of manual contouring is 20 min, that the total median time saved is $7.8 \mathrm{~min}$ when using atlas-based contouring and $10 \mathrm{~min}$ for deep learning contouring. The delineating time is different due to different software and contouring organs. AiContour® @ greatly saves time, because AiContour ${ }^{\circledR}$ (C) is a model established by deep learning. Due to its own database of convolution neural network learning, a good contour of organ at risk is achieved ${ }^{[12]}$. In terms of Raystation $® \bigodot$, the target image and database image deformation registration technology is implemented clinically, that the algorithm is not excellent enough, leading the result of the contour is not always satisfied.

As shown in Fig. 3-8, AiContour ${ }^{\circledR}$ (c) has a relatively concentrated distribution value in the data distribution, and the density of the upper and lower bounds are not much different. When there is obvious difference in density in the automatic

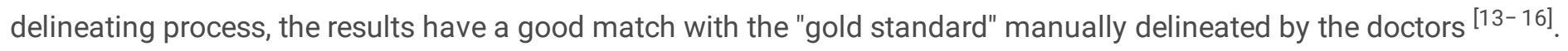
Due to the obvious difference between the density of leg bones and lungs and the density of surrounding tissues, there is not much difference between Raystation $\AA$ (C) and AiContour $\AA$ (C) in the delineation of the leg bones and the bilateral lungs. 
Both areas have reached a comparative level (DSC > 0.7) because of the contrast between bones and soft tissue,which can be used clinically with only partial modification [17]. but in the delineation of the rectum, bladder, stomach, pancreas and other organs, because different patients are affected by own meal and drink. The amount, digestion, and the difference in own physical condition will affect the delineation.. The worst results from the two software were showing in the pancreas. The low contrast in the boundary between the pancrea and the surrounding tissue under ordinary CT scans may cause the boundary not to be defined clearly, resulting in poor results. The $D_{v}$ value of most of the contour results may not be statistically significant due to the difference between the positive and negative data [18].

The spinal cord is contoured in CT images of lung cancer and rectal cancer, and independent samples are tested. The two results are statistically different $(P<0.05)$. The difference between the two groups of data is probably due to the small volume of the spinal cord itself. Individual and statistical differences caused by the data of the contour results are more significant. According to the OI and DSC of results from AiContour ${ }^{\circledR} \subset$, the delineation results of different positions of the spinal cord is relatively close. Although the delineation results are different, the data distribution remains relatively centralized, which shows that AiContour $®($ delineating results are stable. From the theory of auto-contouring, Raystation ${ }^{\circledR}(\mathcal{C}$ needs to match the best case in the database for deformation registration. The U-net network is a CNN (Convolutional Neural Network) -based image segmentation network. It uses a computer to input two-dimensional or threedimensional images to the image pixels. Each image pixel is assigned a weight coefficient and weight quality. By translation and frequency conversion, it is trained to find a feature template suitable for classification, and can quickly filter and match feature pixels on the grid of the image during reading ${ }^{[19-22]}$. Raystation ${ }^{\circledR}\left({ }^{\circ}\right.$, due to the inconsistency of organs as different ages and shapes, it is difficult to establish a universal map. The registration process is deformable, which is time consuming. The accuracy of AiContour $®$ () is based on the raw data of delineating accuracy results, and the output accuracy is not necessarily the optimal solution of the delineating results, and optimization learning is required.

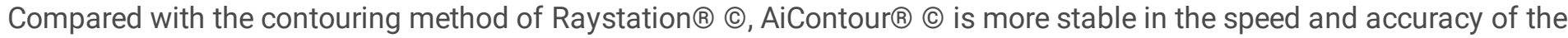
chest and abdomen organs and has certain advantages.

\section{Conclusions}

Both software can be used for automatic contouring. AiContour ${ }^{\circledR}(\subset)$ achieves better contouring results on OAR delineate of lung cancer patients and rectal cancer patients. The volume of chest and abdominal organs is large. Raystation ${ }^{\circledR}\left({ }^{\circ}\right)$ the delineation is good for lung and heart, and the result for the femoral head is good in patients with rectal cancer. However, AiContour ${ }^{\circledR}($ () is better in both scenario and can be used clinically. Both software are not ideal for the contour of the pancreas, so the algorithm of image segmentation needs to be optimized on the less obvious parts of tissues and organs to have two software used in the automatic delineation of chest and abdomen organs. Even if AiContour® () is accepted better clinically, the result of automatic delineating varies from person to person and needs to be identified and corrected by doctors in time.

\section{Declarations}

\section{Acknowledgmens}

Not applicable

\section{Authors' contributions}

CWJ, LYC, conceived the study, participated in its design and coordination and helped draft the manuscript. JYS, generated the manual contours. CWJ,LYC,WC,ZWM ,QLY,YSY and RFF,analyzed parts of the data, and interpreted the data. All authors read and approved the final manuscript. 


\section{Funding}

This research was partially supported by the Zhejiang Basic Public Welfare Research Project $\$ GF21H180053】and the Zhejiang Medical and Health Science and Technology Plan Project $₫ 2021 P Y 002 \rrbracket$

\section{Availability of data and materials}

The data are not available for public access because of patient privacy

concerns, but are available from the corresponding author on reasonable

request.

\section{Ethics approval and consent to participate}

This study was approved by our institutional review board and conducted in accordance with the ethical standards of the Declaration of Helsinki.

\section{Consent for publication}

Not applicable.

\section{Competing interests}

The authors declare that they have no competing interest.

\section{Author details}

1 Department of Radiation Oncology, Zhejiang Provincial People's

Hospital ,Hangzhou 310014 Zhejiang, PR China

2 People's Hospital of Hangzhou Medical College, Hangzhou 310014 Zhejiang, PR China

3 Department of Nuclear Science and Technology, University of South China, Hengyang 421001 Hunan, PR China 4 Department of Radiationtherapy, Shanghai Pulmonary Hospital, 200433 Shanghai, PR China

\section{References}

1. Stein Justin J. Progress in Radiation Therapy[J]. American Journal of Clinical Pathology, 1966(1):1.

2. Bernier J,Hall EJ,GiacciaA.Radiation oncology:a century of achievements.Nat Rev Cancer,2004,4: 737-747.

3. Chao KSC, Bhide S, Chen H, et al. Reduce in Variation and Improve Efficiency of Target Volume Delineation by a Computer-Assisted System Using a Deformable Image Registration Approach[J]. International Journal of Radiation Oncology*Biology*Physics. 2007;68(5):1512-21. 
4. Lin L, Dou Q, Jin YM, et al. Deep learning for automated contouring of primary tumor volumes by MRI for nasopharyngeal carcinoma[J]. Radiology. 2019;291(3):677-86.

5. ANDERS LC, STIELER F, SIEBENLIST K, et al. Performance of an atlas-based autosegmentation software for delineation of target volumes for radiotherapy of breast and anorectal cancer [J]. RadiatOncol. 2012;102(1):68-73.

6. Jeremiah Hwee AV, Louie S, Gaede, et al. Technology Assessment of Automated Atlas Based Segmentation in Prostate Bed Contouring[J]. Radiat Oncol. 2011;6(1):110.

7. Amy VY, Angela W, Iddo W,et al.Atlas-based segmentation improves consistency and decreases time required for contouring postoperative endometrial cancer nodal volumes[J].Int J Radiation oncology biol Phys,2011,79(3): 943947.

8. Ronneberger O, Fischer P, Brox T. U-net: Convolutional networks for biomedical image segmentation[C]//International Conference on Medical image computing and computer-assisted intervention. Springer, Cham, 2015: 234-241.

9. Lustberg T, van Soest J, Gooding M, et al. Clinical evaluation of atlas and deep learning based automatic contouring for lung cancer[J]. Radiother Oncol. 2018;126(2):312-7.

10. Nelms BE Tomé, WA,Robinson G,et al. Variations in the contouring of organs at risk:test case from a patient with oropharyngealcancer[J]. Int J Radiat Oncol Biol Phys. 2012;82(1):368-78.

11. Lustberg T, Soest JV, Gooding M, et al. Clinical evaluation of atlas and deep learning based automatic contouring for lung cancer[J]. Radiother Oncol. 2017;126(2):312-7.

12. Young AV, Wortham A, Wernick I, et al. Atlas-Based Segmentation Improves Consistency and Decreases Time Required for Contouring Postoperative Endometrial Cancer Nodal Volumes[J]. 79(3):943-947.

13. Hardcastle N, Van Elmpt W, De Ruysscher D, et al. Accuracy of deformable image registration for contour propagation in adaptive lung radiotherapy[J]. Radiat Oncol. 2013;8(1):243.

14. Deeley MA, Chen A, Datteri RD, et al. Segmentation editing improves efficiency while reducing inter-expert variation and maintaining accuracy for normal brain tissues in the presence of space-occupying lesions[J]. Phys Med Biol. 2013;58(12):4071.

15. Velker VM, Rodrigues GB, Dinniwell R, et al. Creation of RTOG compliant patient CT-atlases for automated atlas based contouring of local regional breast and high-risk prostate cancers[J]. Radiat Oncol. 2013;8(1):188.

16. Valentini V, Boldrini L, Damiani A, et al. Recommendations on how to establish evidence from auto-segmentation software in radiotherapy[J]. Radiother Oncol. 2014;112(3):317-20.

17. ZIJDENBOS A P, DAWANT B M MARGOLINRA, et alMorphometric analysis of white matter lesions in MR images: methodand validation [J]. IEEE Trans Med Imaging, 1994, 13(4): 716-724.Valentini V, Boldrini L, Damiani A, et al. Recommendations on how to establish evidence from auto-segmentation software in radiotherapy[J]. Radiotherapy and Oncology, 2014, 112(3): 317-320.

18. Khawandanh E, Mcvicar N, Duzenli C. Poster - 37: Pre-clinical geometric, dosimetric and timing assessment of head and neck OARs using an in-house atlas-based auto-segmentation (ABAS) tool[J]. Med Phys. 2016;43(8):4944-5.

19. Hao X, Zhang G, Ma S. Deep Learning[J]. International Journal of Semantic Computing. 2016;10(03):417-39.

20. Men K, Dai J, Li Y. Automatic segmentation of the clinical target volume and organs at risk in the planning CT for rectal cancer using deep dilated convolutional neural networks[J]. Medical physics. 2017;44(12):6377-89.

21. Gu J, Wang Z, Kuen J, Ma L, Shahroudy A, Shuai B, Liu T, Wang X, Wang L, Wang G, Cai J. 2015. Recent advances in convolutional neural networks. arXiv preprint arXiv:1512.07108.

22. Branson S, Van Horn G, Belongie S, et al. Bird species categorization using pose normalized deep convolutional nets[J]. arXiv preprint arXiv:1406.2952, 2014.

\section{Figures}




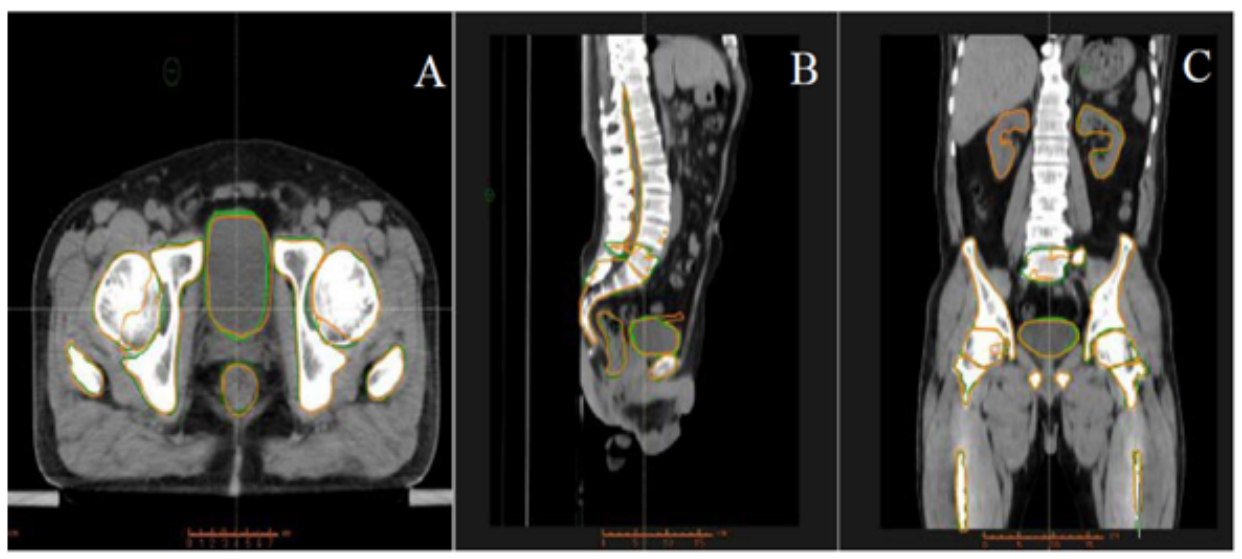

AiContour ${ }^{\circledR}$

Manual contour

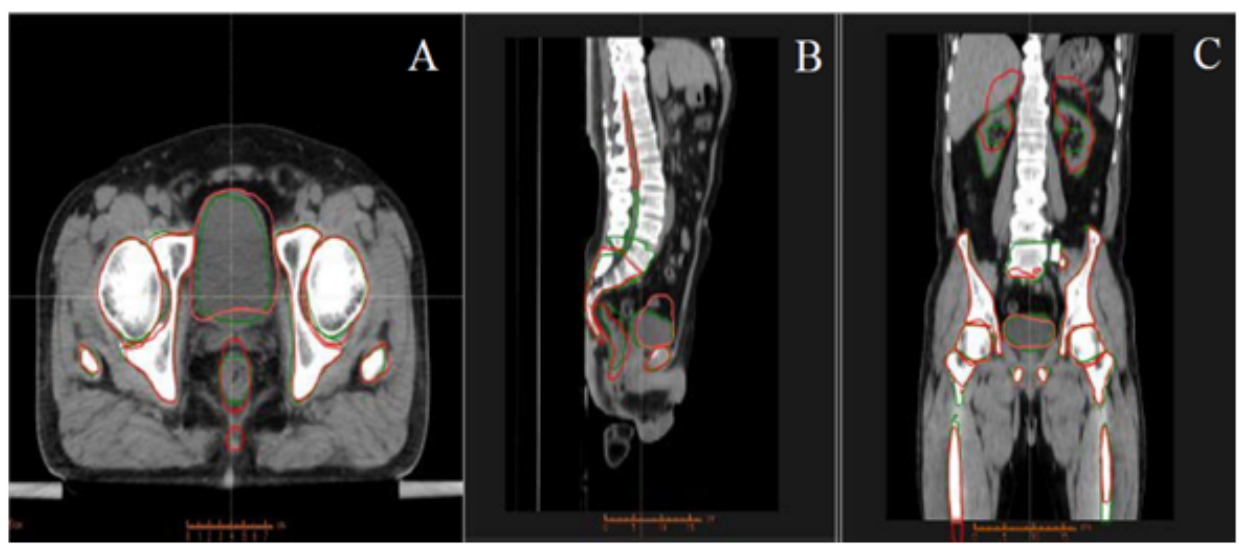

Figure 1

Comparison of OAR results of rectal cancer patients using two software ( $\mathrm{A}$ is the traverse plane, $\mathrm{B}$ is the sagittal plane, $\mathrm{C}$ is the coronal plane) 

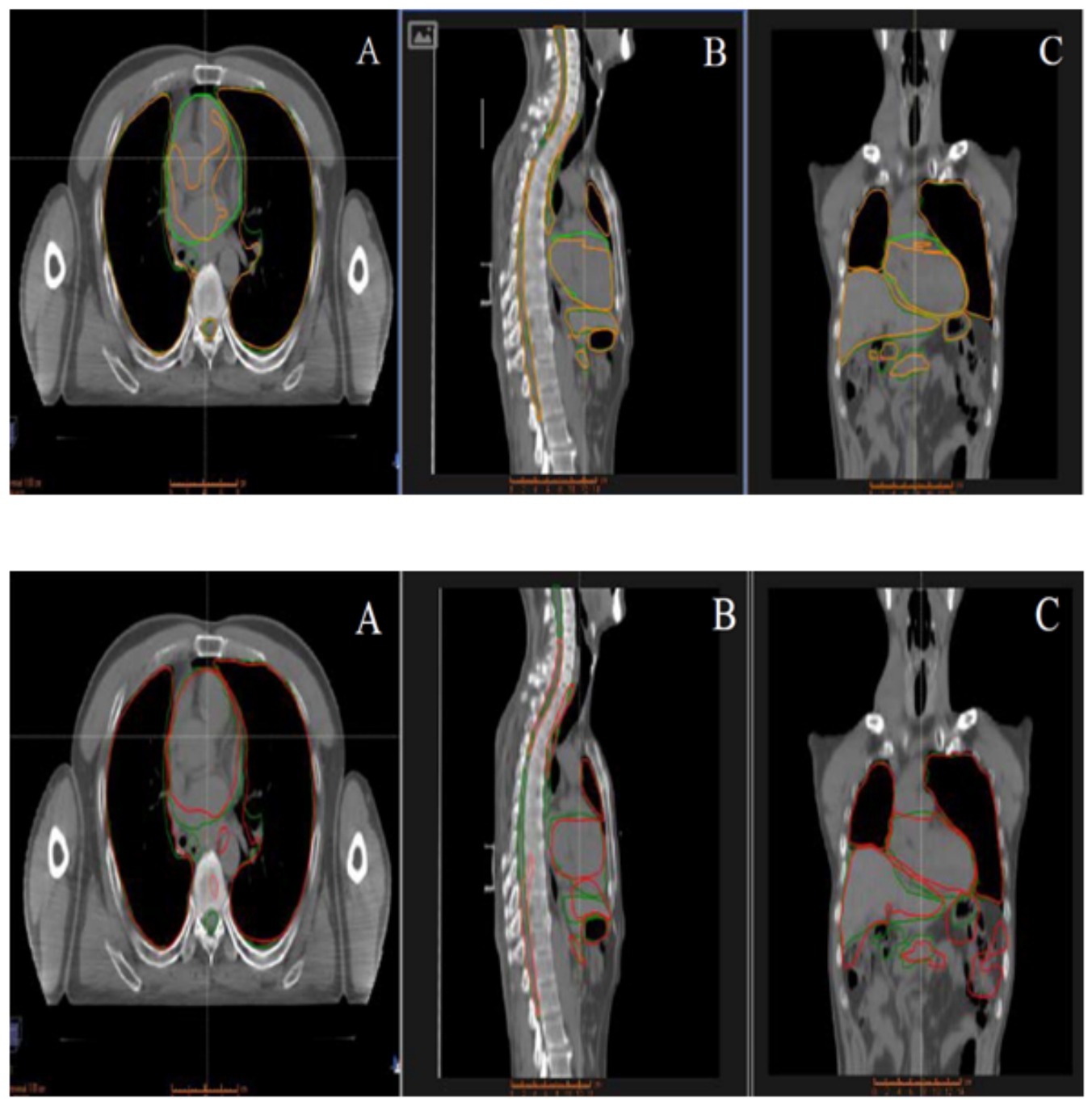

Figure 2

Comparison of OAR results of lung cancer patients using two software ( $\mathrm{A}$ is the traverse plane, $\mathrm{B}$ is the sagittal plane, $\mathrm{C}$ is the coronal plane) 


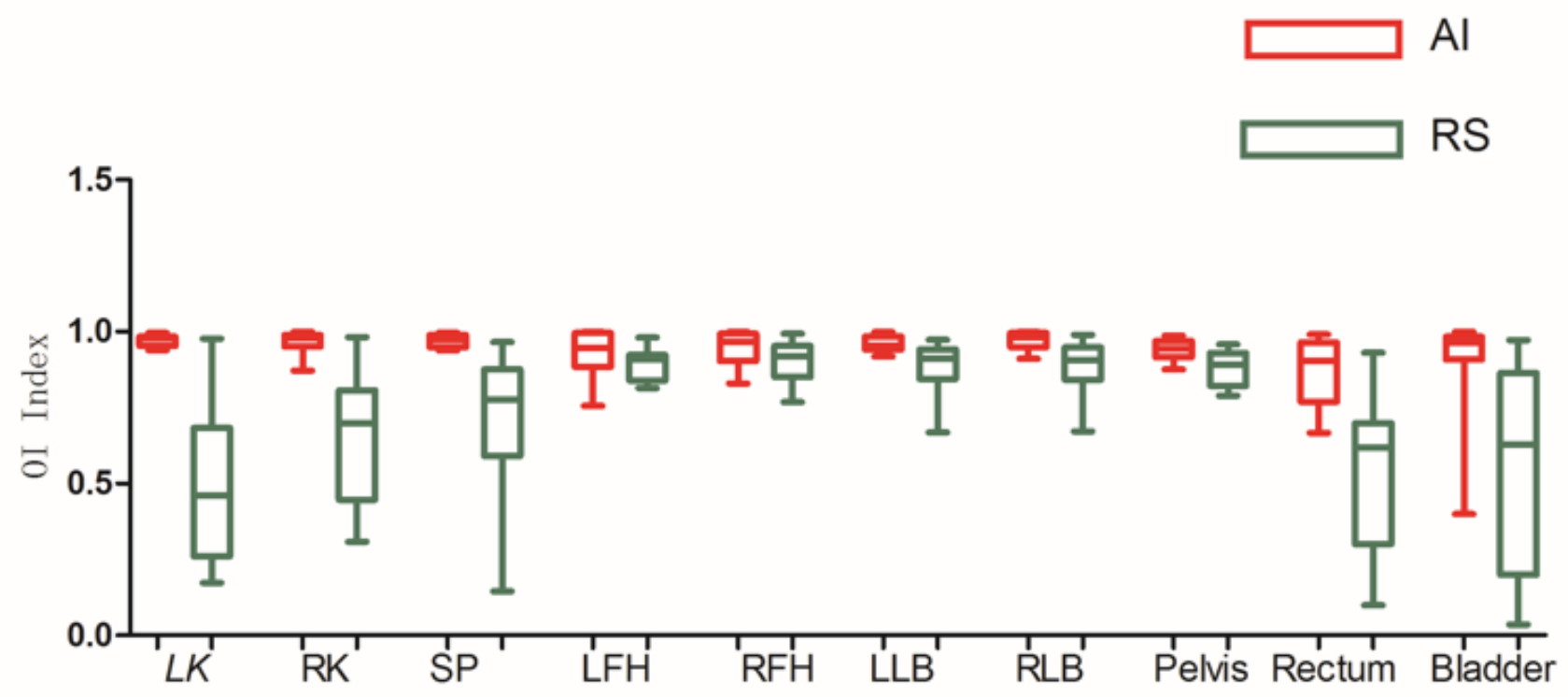

Figure 3

Comparison results of OI between two software in automatic delineation of rectal cancer OAR Notation: LK means left kidney,RK means right kidney, SP means spinal cord,LFH means left femoral head,RFH means right femoral head,LLB means left leg bone, RLB means right leg bone囚

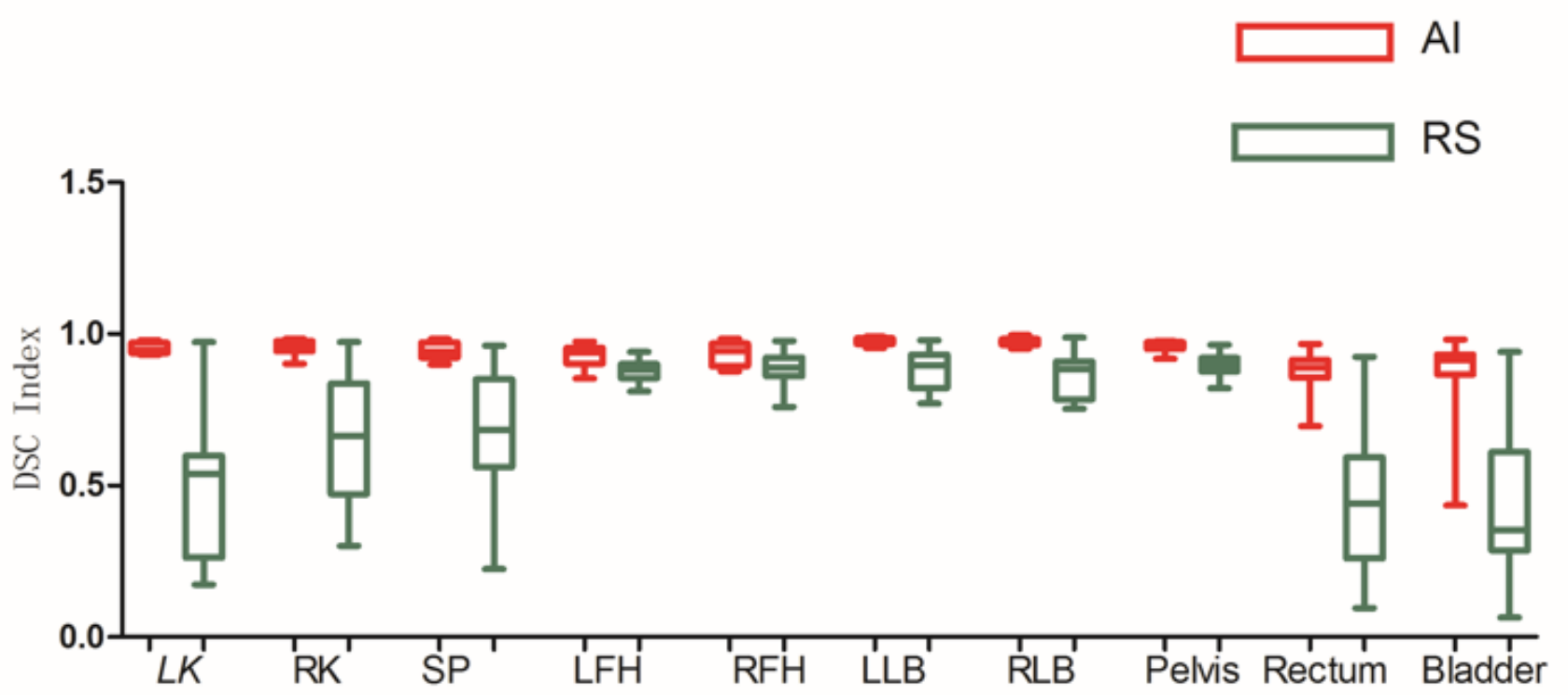

Figure 4

Comparison results of DSC between two software in automatic delineation of rectal cancer OAR 


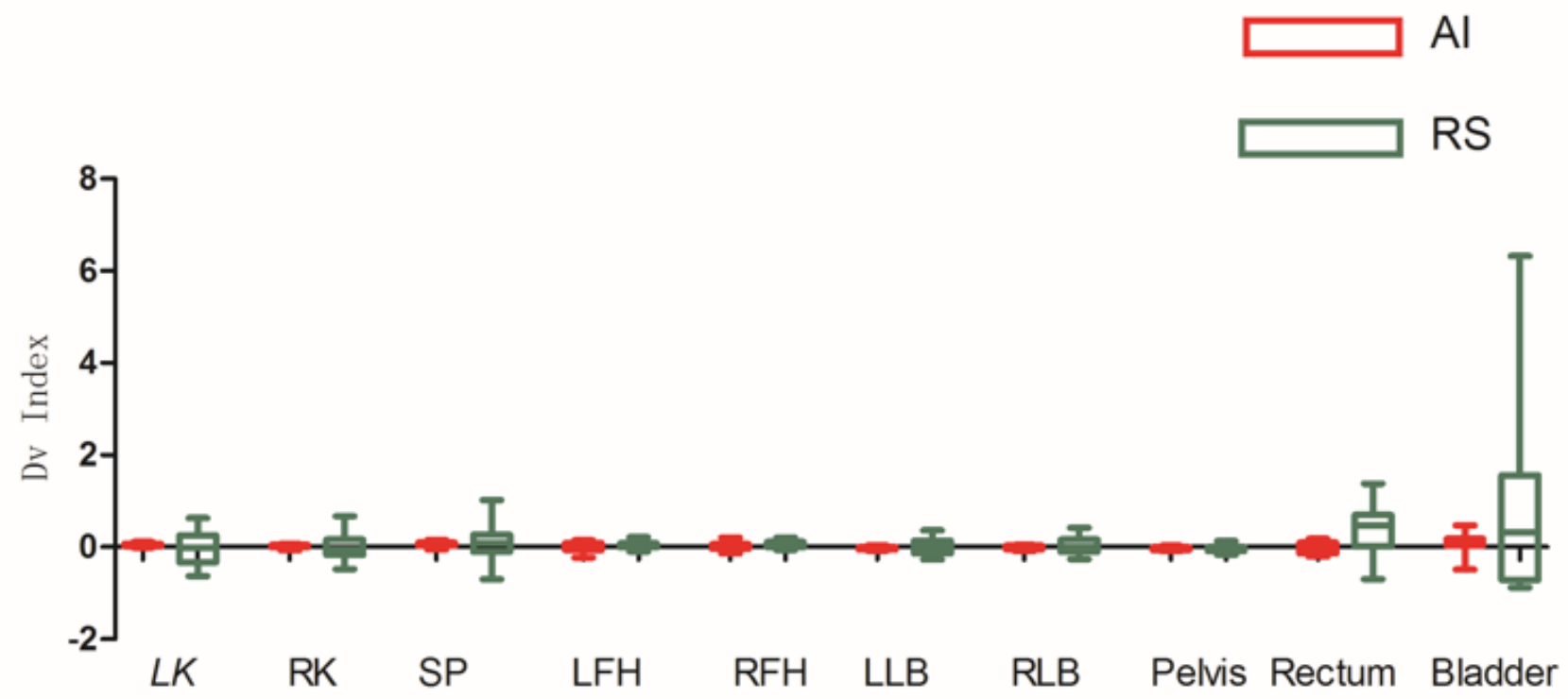

Figure 5

Comparison results of Dv between two software in automatic delineation of rectal cancer OAR

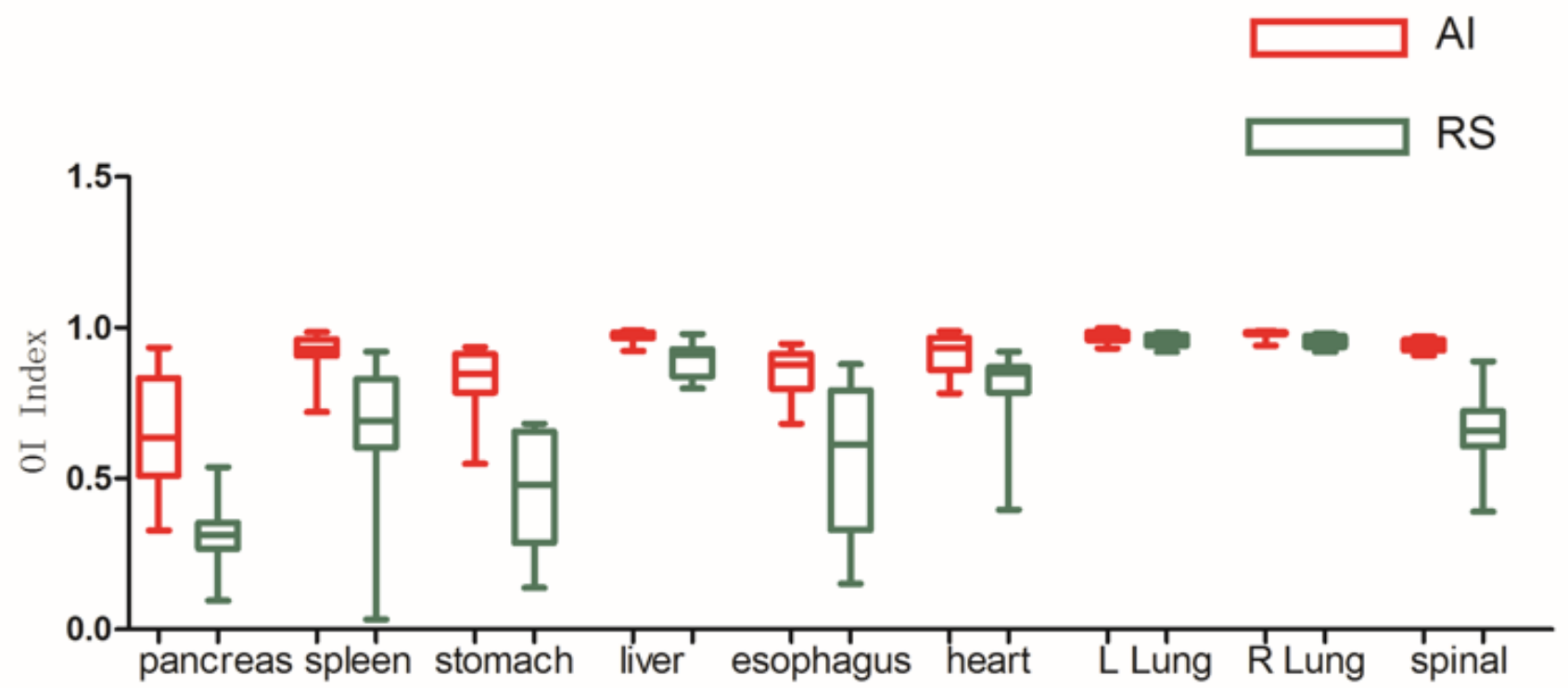

Figure 6

Comparison results of OI between two software in automatic delineation of lung cancer OAR 


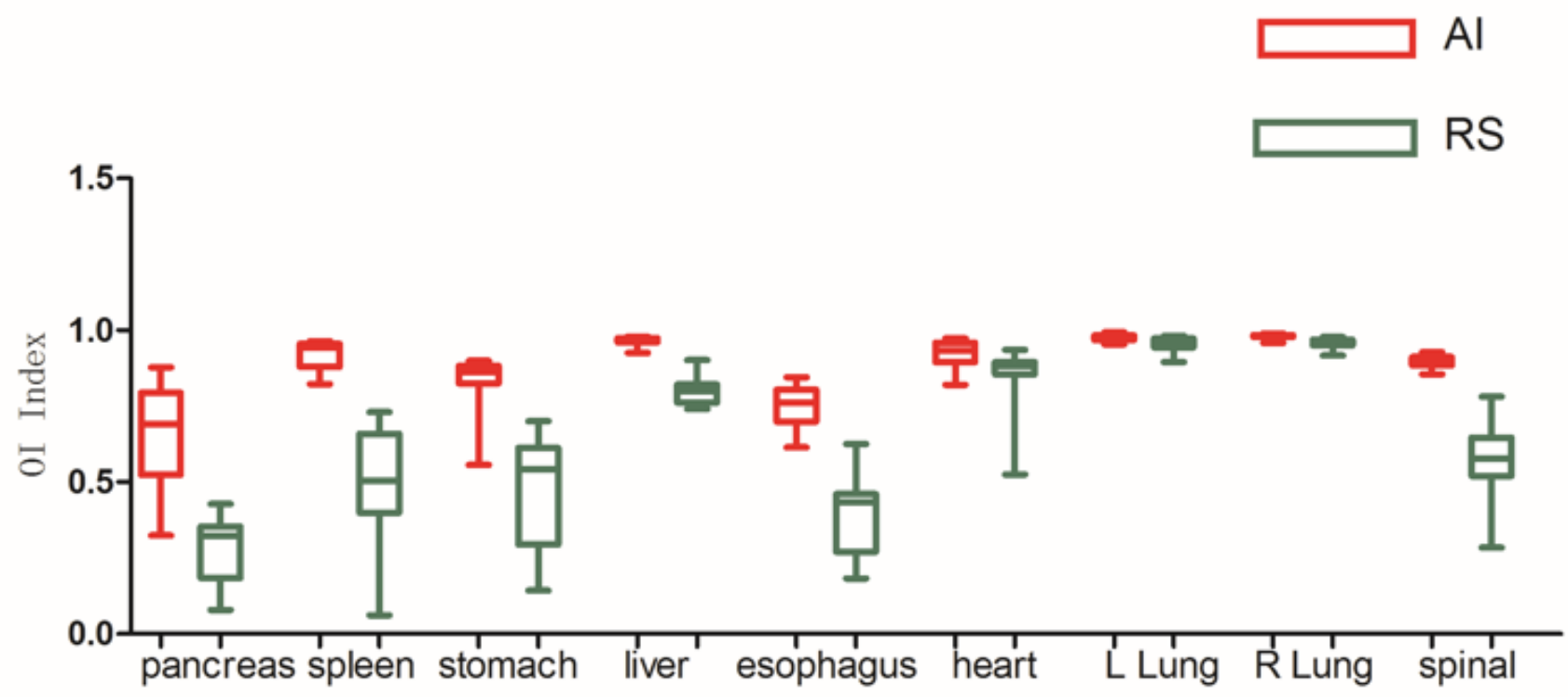

Figure 7

Comparison results of DSC between two software in automatic delineation of lung cancer OAR

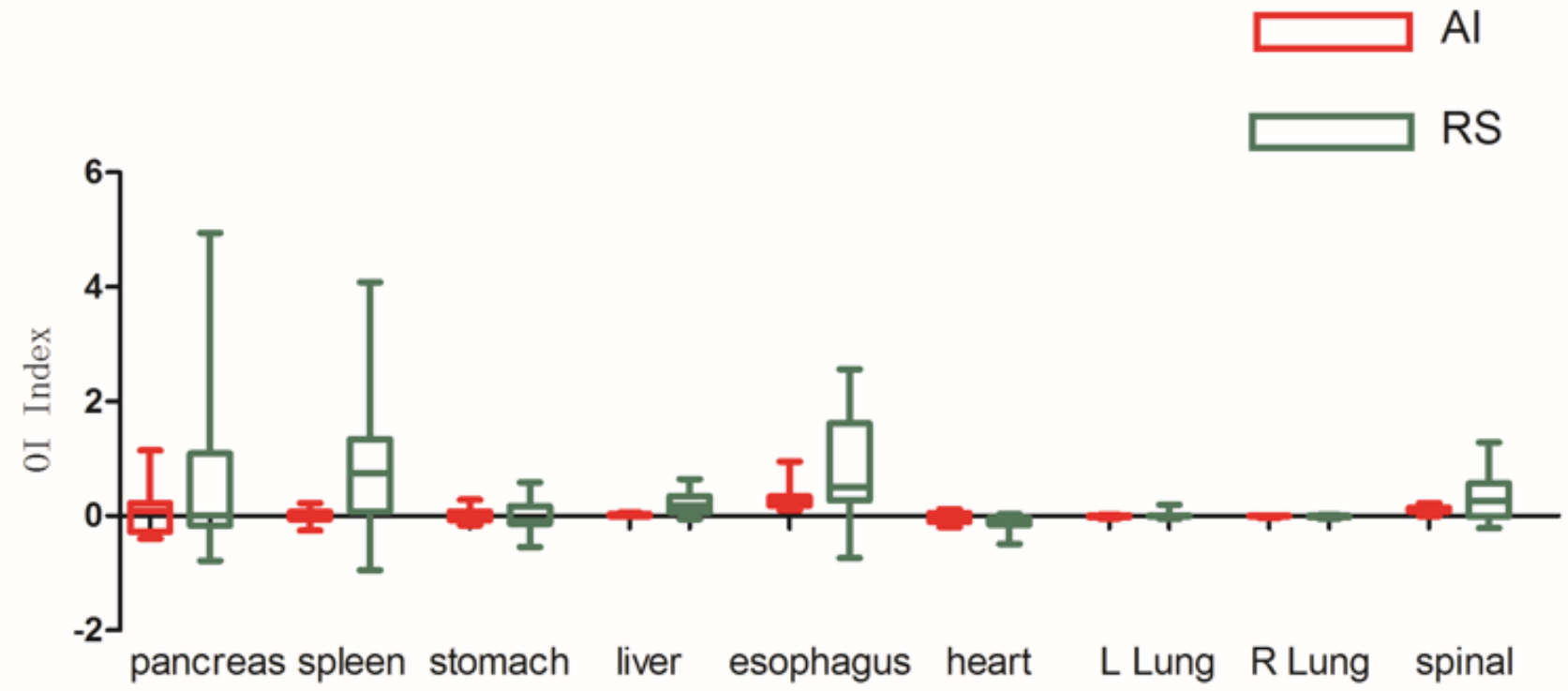

Figure 8

Comparison results of Dv between two software in automatic delineation of lung cancer OAR 